\title{
Hepatic epithelioid hemangioendothelioma (HEHE) - rare vascular malignancy mimicking cholangiocarcinoma: a case report
}

\author{
Michal Kawka^, Sau Mak, Shengyang Qiu, Tamara M. H. Gall, Long R. Jiao \\ HPB Surgical Unit, Department of Surgery \& Cancer, Imperial College London, Hammersmith Hospital Campus, Du Cane Road, London, UK \\ Correspondence to: Mr. Shengyang Qiu. HPB Surgical Unit, Department of Surgery \& Cancer, Imperial College London, Hammersmith Hospital \\ Campus, Du Cane Road, London, W12 0HS, UK. Email: s.qiu@imperial.ac.uk.
}

\begin{abstract}
Hepatic epithelioid hemangioendothelioma (HEHE) is a rare, often misdiagnosed malignancy of vascular origin. We describe a case of a 36-year-old, who presented with a burning sensation in his chest on exercise and was believed to have bilobar intrahepatic cholangiocarcinoma. After receiving chemotherapy with partial response, the patient underwent staged resection with modified associating liver partition with portal vein ligation for staged hepatectomy (ALPPS) procedure-laparoscopic radiofrequencyassisted ALPPS (RALPPS). Histological examination of the stage 1 specimen revealed HEHE deposits in the left lobe of the liver. The patient proceeded to have stage 2 open right hepatectomy with concurring histology. Ninety-day follow-up computed tomography scan showed almost complete resolution of HEHE lesions. Low incidence, non-specific clinical and radiological characteristics all contribute to high HEHE misdiagnosis rate. Histological analysis can be used for confirmation of the diagnosis; however, specialist staining is required, which is not routinely performed. Multiple treatments are available against HEHE, but only liver resection and liver transplantation are potentially curative. ALPPS and its modifications (such as RALPPS) offer an alternative from conventional two-stage hepatectomy for patients with extensive bilobar HEHE, who are at risk of developing post-hepatectomy liver failure or patients with anomalous hepatic vasculature anatomy which makes portal vein embolisation (PVE) challenging.
\end{abstract}

Keywords: Associating liver partition and portal vein ligation for staged hepatectomy (ALPPS); epithelioid hemangioendothelioma; minimally invasive surgery; hepatic tumours; case report

Received: 27 September 2020; Accepted: 07 January 2021; Published: 25 October 2022.

doi: $10.21037 /$ tgh-20-310

View this article at: http://dx.doi.org/10.21037/tgh-20-310

\section{Introduction}

Hepatic epithelioid hemangioendothelioma (HEHE) is a rare vascular malignancy (incidence $<0.1$ per 100,000 people) (1). It is a low-grade vascular neoplasm exhibiting behaviour between that of haemangioma and angiosarcoma. However, due to its atypical presentation, and unpredictable clinical course, there is no standardised management pathway for such tumours.

This case highlights the diagnostic and therapeutical challenges of HEHE, including an atypical presentation and inconclusive radiological findings. Due to extensive bilobar disease, we have decided to use a unique management strategy, for HEHE, a modification of conventional associating liver partition with portal vein ligation for staged hepatectomy (ALPPS) technique, which is, to the best of our knowledge, the first of its kind. We present the following article in accordance with the CARE reporting checklist (available at https://tgh.amegroups.com/article/ view/10.21037/tgh-20-310/rc).

\section{Case presentation}

A 36-year-old man presented in July 2018 complaining of

\footnotetext{
$\wedge$ ORCID: 0000-0002-5826-8355.
} 


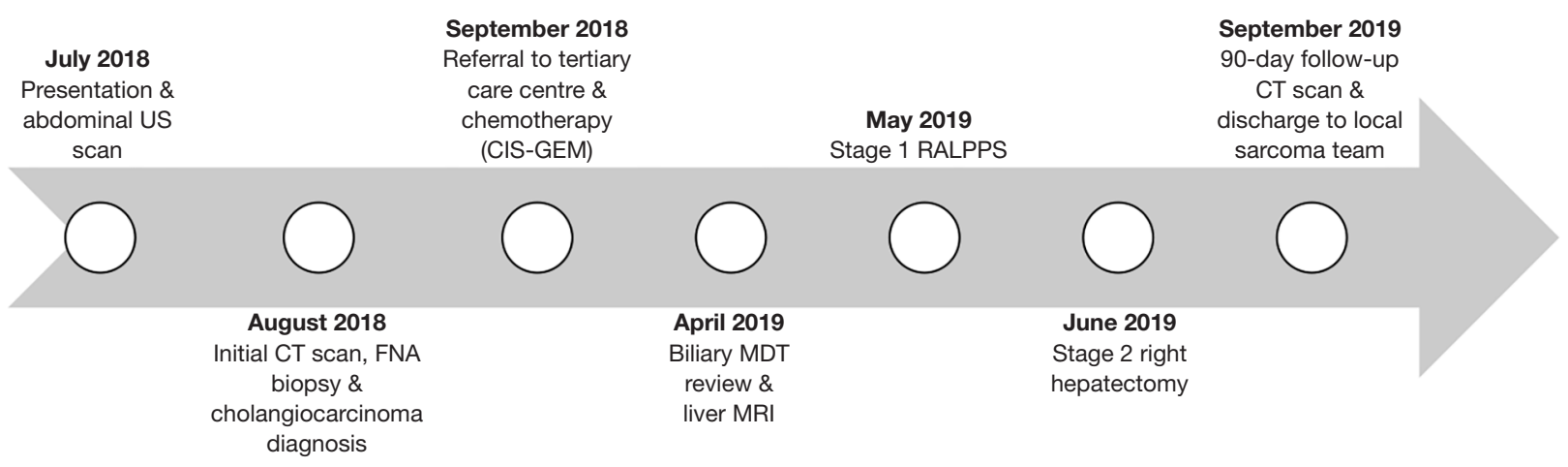

Figure 1 Timeline of the events. US, ultrasound scan; CT, computed tomography; FNA, fine needle aspiration; CIS-GEM, cisplatingemcitabine; MRI, magnetic resonance imaging; MDT, multi-disciplinary team; RALPPS, radiofrequency-assisted associating liver partition and portal vein ligation for staged hepatectomy.

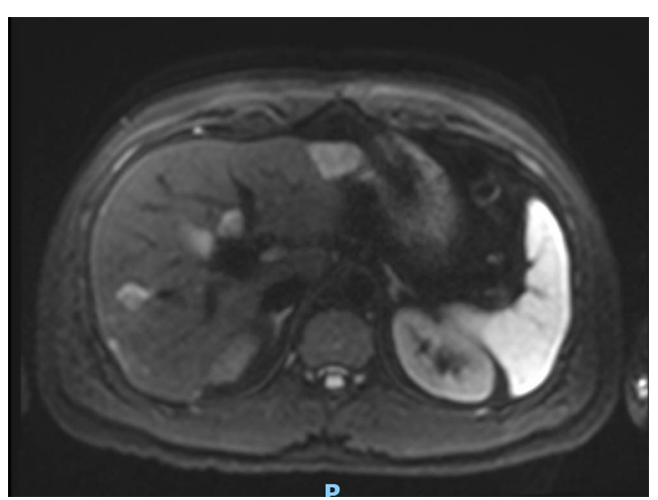

Figure 2 MRI scan of the liver after presentation. MRI, magnetic resonance imaging.

a burning sensation in his chest exacerbated by exercise (Figure 1). At the time of presentation, the patient had no notable past medical history, family history or associated complaints. However, in 2016 the patient complained of loose stools and reflux for an unspecified amount of time, but no diagnosis was established following a colonoscopy and an oesophagogastroduodenoscopy in 2016.

The initial physical examination of the major systems, including an abdominal examination was unremarkable. The patient was not jaundiced and did not complain of abdominal pain or discomfort. However, in light of the previous medical history and lack of associated cardiac or respiratory complaints, a routine abdominal ultrasound was conducted and discovered a mass in the left lobe of the liver. Computed tomography (CT) scan of the thorax, abdomen and pelvis confirmed multiple irregular hypoattenuating lesions throughout both lobes of the liver, with no intrahepatic biliary duct dilatation. No extrahepatic nodal disease was found.

Ultrasound-guided fine needle aspiration (FNA) biopsy of the left lobe lesion showed a moderately differentiated adenocarcinoma. The tumour was CK7 positive, CK20 negative, chromogranin/TTF1/CDX2 negative and prostate-specific antigen (PSA)/heat stable antigen (HSA) negative. Upon impression of adenocarcinoma liver metastases, fluorodeoxyglucose (FDG) positron emission tomography (PET) was carried out to exclude occult primary site of malignancy-liver lesions were found to be modestly FDG avid, however, no occult primary was identified. The investigation results were reviewed by a multidisciplinary team (MDT), and the most likely diagnosis was felt to be a primary intrahepatic cholangiocarcinoma with multiple bilobar liver lesions.

The patient was referred to the medical oncology team at our tertiary care centre in September 2018. Hepatic tumour markers were unremarkable at the time of referral [carcinoembryonic antigen $(\mathrm{CEA})=1.3 \mathrm{ng} / \mathrm{mL}$, alphafetoprotein $(\mathrm{AFP})=3.6 \mathrm{ng} / \mathrm{mL}$, cancer antigen 19-9 (CA19-9) $=3 \mathrm{U} / \mathrm{mL}$, human chorionic gonadotropin $(\mathrm{HCG})<0.1 \mathrm{IU} / \mathrm{mL}]$. Based on initial findings and the working diagnosis of cholangiocarcinoma, the patient was managed by cisplatingemcitabine (CIS-GEM) neo-adjuvant chemotherapy.

The case was reviewed in a specialist hepatobiliary MDT during the ninth cycle of CIS-GEM chemotherapy in April 2019. A magnetic resonance imaging (MRI) with contrast (Figure 2) was scheduled and revealed a $33-\mathrm{mm}$ mass in the left lobe and multiple focal lesions (maximum $15 \mathrm{~mm}$ ) throughout the right lobe. All lesions demonstrated low signal on $\mathrm{T} 1$ and high signal on $\mathrm{T} 2$ contrast weighting. The 


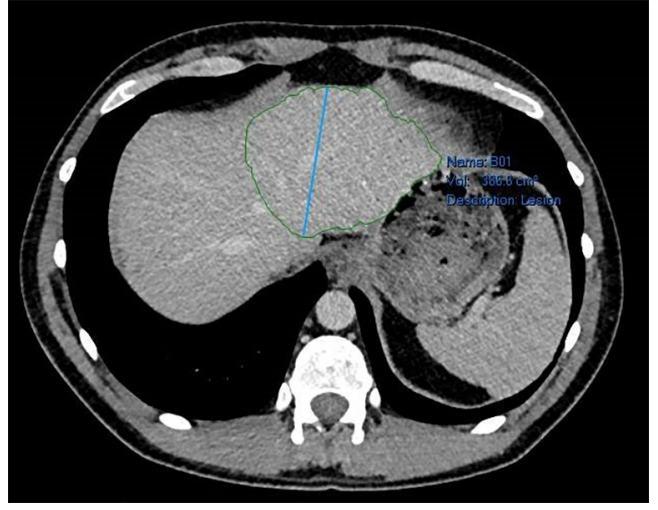

Figure 3 CT scan of the liver after stage 1 of RALPPS showing FLR. CT, computed tomography; RALPPS, radiofrequencyassisted associating liver partition and portal vein ligation for staged hepatectomy; FLR, future liver remnant.

33-mm left lobe lesion exhibited minor peripheral arterial enhancement with gradual filling from the periphery on both portal venous and 5-minute phase. Right lobe lesions showed no enhancement. There was an overall positive response to treatment, with all deposits decreasing in size compared to baseline and no evidence of new intrahepatic or extrahepatic disease (partial response by RECIST criteria) (2). There remained bilobar disease involvement (more marked in the right lobe of the liver) with no evidence of portal vein thrombosis, and appropriately enhancing hepatic veins.

As the patient was young, had a good pre-operative performance status and normal liver function, in light of extensive bilobar disease, it was agreed that the patient was suitable for two-stage hepatectomy. Portal vein embolisation (PVE) was considered as an option, however, due to variant hepatic vascular anatomy (early bifurcation of the anterior and posterior sectoral branch of the right hepatic vein), it was considered challenging and decided against. Instead, portal vein ligation was the technique of choice, diverting away from classical two-stage hepatectomy in favour ALPPS. Stage 1 was a modification of conventional ALPPS-laparoscopic radiofrequency-assisted ALPPS (RALPPS), followed by open stage 2 right hepatectomy, both techniques previously as previously described by the senior author LR Jiao $(3,4)$.

Laparoscopic RALPPS was carried out along with wedge resection of a segment 3 lesion and multiple radiofrequency ablations (RFA) of left lobe lesions in May 2019 without complications. Re-staging CT abdomen and

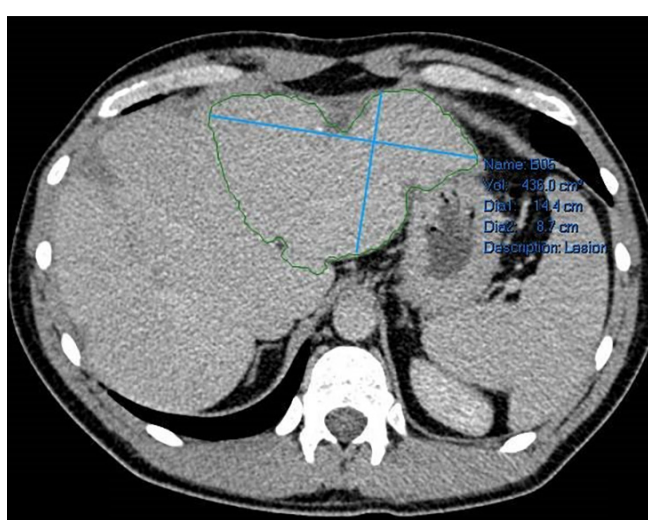

Figure 4 CT scan of the liver before stage 2 of RALPPS showing FLR hypertrophy. CT, computed tomography; RALPPS, radiofrequency-assisted associating liver partition and portal vein ligation for staged hepatectomy; FLR, future liver remnant.

pelvis with contrast was performed 2 weeks later. Future liver remnant (FLR) volume increased from 386 to $436 \mathrm{~cm}^{3}$ ( $13 \%$ increase), and as such was deemed satisfactory for progression to stage 2 (Figures 3,4).

The histological specimen was evaluated. Macroscopically, a pale, oval, and indurated capsular lesion ( $\varnothing 26 \mathrm{~mm}$ ) was found in segment 3 specimen. Microscopically, invasion around preserved portal tracts and central veins was observed. The neoplasm was composed of epithelioid and stellate cells, with variable pink cytoplasm embedded in a hyaline sclerotic stroma and poorly formed vascular structures were seen peripherally. Immunohistochemistry of the tumour was positive for CD31 and CD34. Therefore, a histological diagnosis of HEHE was established.

Despite a diagnosis of HEHE, given the number of lesions in the right lobe, we proceeded to open the right hepatectomy as planned (4 weeks interval), again without immediate complications. Histopathology of stage 2 specimens reported multiple deposits of HEHE with vascular invasion, all of which were consistent with those in the left lobe.

Follow-up CT scan at 90 days showed good response to surgical treatment, with almost complete resolution of HEHE lesions and no evidence of distant disease (Figure 5). The patient developed a collection in the gallbladder fossa proximity, believed to be a biloma, and thus was referred to interventional radiology for percutaneous drainage. Few mildly enlarged lymph nodes were noted adjacent to the gallbladder collection. The patient was transferred to a specialist sarcoma team for the continuation of his 


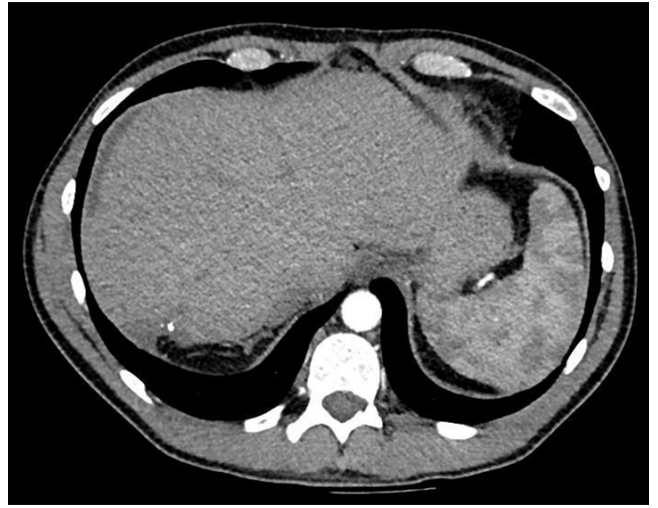

Figure 5 CT scan of the liver after stage 2 of RALPPS. CT, computed tomography; RALPPS, radiofrequency-assisted associating liver partition and portal vein ligation for staged hepatectomy.

oncological care and regular surveillance scanning for the next 5 years.

All procedures performed in this study were in accordance with the ethical standards of the institutional and/or national research committee(s) and with the Helsinki Declaration (as revised in 2013). Written informed consent was obtained from the patient for publication of this case report and accompanying images. A copy of the written consent is available for review by the editorial office of this journal.

\section{Discussion}

HEHE is often overlooked as a differential diagnosis for liver lesions. A previous literature review identified that $60-80 \%$ of HEHE patients are initially misdiagnosed, most commonly with cholangiocarcinoma, angiosarcoma or hepatocellular carcinoma (5). The extremely high misdiagnosis rate is likely to be multifactorial, as low incidence, atypical presentation, non-conclusive imaging and specialist histopathology stains requirements all contribute to possible initial diagnostic mistake.

At presentation, patients with HEHE report nonspecific symptoms such as right upper quadrant pain, upper abdominal discomfort, weight loss, back pain or malaise; however, up to $25 \%$ are asymptomatic. A minority of patients present with a clinical picture consistent with the hepatic origin of the disease (6). HEHE is more prevalent in women than men (3:2 ratio), with the mean age at diagnosis being 42 years (6).

Although multiple imaging modalities can assist in distinguishing HEHE from other hepatic masses, MRI is the most useful one. The majority of HEHE are multifocal, characterised by peripheral location, often extending to the liver capsule with retraction (7). On MRI, HEHE show low signal intensity in T1-weighted images, while showing high signal intensity and characteristic "target-like" appearance (a central high signal with peripheral halo) in T2-weighted images (8). On the hepatobiliary phase, HEHE may exhibit the "lollipop-sign" (hepatic/portal vein tapering at the margin of a defined hypoenhancing lesion) (7). Although these signs can assist in diagnosis, hepatobiliary radiological expertise and clinical correlation are required to correctly identify HEHE based on imaging alone. As such, HEHE is often mistakenly identified as other tumours with a higher incidence and similar imaging characteristics (hepatocellular carcinoma, angiosarcoma or cholangiocarcinoma-as in the case described).

Due to the ambiguity of information provided by imaging, the diagnosis of HEHE can only be confirmed via histological examination. Macroscopically, HEHE is typically a firm, tan tumour. Microscopically, it shows a characteristic zonal pattern of cellularity (hypercellular periphery and hypocellular centre with cartilaginous transition zone). Moreover, lobular architecture remains intact, with portal tract remnants preserved. The majority of HEHE tumours stain positively for factor-VIII related antigen, as well as endothelial cell markers (CD31 and CD34), which is consistent with the vascular origin of the neoplasm (9). Negative staining for markers of other hepatic malignancies (bile, mucin, CEA, AFP) can aid in diagnosis (10). However, these immunohistochemical stains require an existing working diagnosis of HEHE, as they are not routinely performed when analysing liver tissue biopsies. In the case we described, these stains were not performed until the post-stage 1 specimen analysis, highlighting the limitation of the diagnostic pathway for HEHE.

Multiple therapeutic modalities can be used to treat HEHE, but due to the unpredictable clinical course of the disease, a standard management pathway is yet to be established (6). Chemotherapy, radiotherapy and immunotherapy are amongst therapies used for management of HEHE; however, it is difficult to comprehensively assess their effectiveness as evidence for their effectiveness is limited due to low incidence $(11,12)$. Moreover, inhibition of angiogenesis in tumour via interference with the vascular endothelial growth factor (VEGF) pathway has been suggested as a potential treatment (13).

Liver transplantation and liver resection are the 
only potentially curative options (14). In some cases, transarterial chemoembolisation (TACE) is utilised either as a monotherapy or as an adjuvant to liver resection (15). The radicality of resection is crucial, as HEHE can respond aggressively to incomplete resection margins, due to liver regeneration mediators stimulating tumour growth (16). Unfortunately, such radical resections may not always be possible as estimated future live remnant (FLR) is insufficient and possibly leading to post-hepatectomy liver failure.

In our case, bilobar disease involvement added another consideration for the choice of treatment. Potential options included hepatectomy with pre-operative PVE, conventional two-stage hepatectomy and staged hepatectomy with ALPPS. Due to variant hepatic vascular anatomy, it was decided collaboratively with the interventional radiology team that PVE would be challenging and unlikely to be successful. Moreover, due to its potentially higher capacity for increasing FLR volume, ALPPS was preferred over conventional two-stage hepatectomy.

Our team has implemented a modification of conventional ALPPS technique-RALPPS, which was performed laparoscopically for added benefits of minimallyinvasive surgery over its open counterparts $(3,4)$. RALPPS is especially effective for patients with bilobar disease, who have the majority of the tumours located in one of the lobes, as it allows for clearance of FLR of any malignancies and subsequent removal of the infiltrated lobe, while maximally preserving the functional parenchyma.

To the best of our knowledge, this is only the fifth reported case of ALPPS performed for a patient with HEHE, but the first of laparoscopic RALPPS. The results of the previous four cases, combined with our experience, are promising and provide initial evidence of ALPPS being feasible and safe for HEHE patients (17-20). However, due to its high complication rate and mortality, ALPPS should only be performed in specialist hepato-pancreato-biliary centres with strict patient selection criteria, for a subgroup of patients who can benefit from the procedure.

It is also worth noting the diagnostic challenges that we encountered. The patient was initially mis-diagnosed with bilobar intrahepatic cholangiocarcinoma, and the diagnosis of HEHE was only made on the histological examination of stage 1 resection specimen. The diagnosis was agreed upon during the MDT discussions based on from the FNA biopsy results, which has shown moderately differentiated adenocarcinoma. However, the relatively young age of the patient, lack of jaundice and other associated biliary complaints and overall good performance status raised suspicion for a potentially different diagnosis. As such, this case highlights the importance of in including HEHE amongst differential diagnoses for patients with extensive bilobar liver lesions who do not fit the clinical picture of cholangiocarcinoma, hepatocellular carcinoma or liver metastases. Such patients should undergo liver MRI (and not only CT as initially happening in our case), as well as having the specialist immunohistochemical staining (factor-VIII related antigen, CD31 and CD34) of biopsy or resection specimen, to ensure the diagnosis of HEHE is not missed.

The initial misdiagnosis and subsequent treatment highlight the difficulty in HEHE management. RALPPS provides an alternative for HEHE patients who cannot undergo traditional liver resection due to extensive tumour burden, have anomalous portal vein anatomy precluding PVE or cannot receive liver transplantation.

\section{Acknowledgments}

Funding: None.

\section{Footnote}

Reporting Checklist: The authors have completed the CARE reporting checklist. Available at https://tgh.amegroups.com/ article/view/10.21037/tgh-20-310/rc

Conflicts of Interest: All authors have completed the ICMJE uniform disclosure form (available at https://tgh.amegroups. com/article/view/10.21037/tgh-20-310/coif). The authors have no conflicts of interest to declare.

Ethical Statement: The authors are accountable for all aspects of the work in ensuring that questions related to the accuracy or integrity of any part of the work are appropriately investigated and resolved. All procedures performed in this study were in accordance with the ethical standards of the institutional and/or national research committee(s) and with the Helsinki Declaration (as revised in 2013). Written informed consent was obtained from the patient for publication of this case report and accompanying images. A copy of the written consent is available for review by the editorial office of this journal.

Open Access Statement: This is an Open Access article distributed in accordance with the Creative Commons 
Attribution-NonCommercial-NoDerivs 4.0 International License (CC BY-NC-ND 4.0), which permits the noncommercial replication and distribution of the article with the strict proviso that no changes or edits are made and the original work is properly cited (including links to both the formal publication through the relevant DOI and the license). See: https://creativecommons.org/licenses/by-nc-nd/4.0/.

\section{References}

1. Hertl M, Cosimi AB. Liver transplantation for malignancy. Oncologist 2005;10:269-81.

2. Eisenhauer EA, Therasse P, Bogaerts J, et al. New response evaluation criteria in solid tumours: revised RECIST guideline (version 1.1). Eur J Cancer 2009;45:228-47.

3. Gall TM, Sodergren MH, Frampton AE, et al. Radiofrequency-assisted Liver Partition with Portal vein ligation (RALPP) for liver regeneration. Ann Surg 2015;261:e45.

4. Jiao LR, Fajardo Puerta AB, Gall TMH, et al. Rapid Induction of Liver Regeneration for Major Hepatectomy (REBIRTH): A Randomized Controlled Trial of Portal Vein Embolisation versus ALPPS Assisted with Radiofrequency. Cancers (Basel) 2019;11:302.

5. Mehrabi A, Kashfi A, Fonouni H, et al. Primary malignant hepatic epithelioid hemangioendothelioma: a comprehensive review of the literature with emphasis on the surgical therapy. Cancer 2006;107:2108-21.

6. Makhlouf HR, Ishak KG, Goodman ZD. Epithelioid hemangioendothelioma of the liver: a clinicopathologic study of 137 cases. Cancer 1999;85:562-82.

7. Epelboym Y, Engelkemier DR, Thomas-Chausse F, et al. Imaging findings in epithelioid hemangioendothelioma. Clin Imaging 2019;58;59-65.

8. Lv P, Lin J. MRI findings of a hepatic epithelioid hemangioendothelioma. Quant Imaging Med Surg 2012;2:237-8.

9. Studer LL, Selby DM. Hepatic Epithelioid Hemangioendothelioma. Arch Pathol Lab Med 2018;142:263-7.

10. Ishak KG, Sesterhenn IA, Goodman ZD, et al. Epithelioid hemangioendothelioma of the liver: a clinicopathologic and follow-up study of 32 cases. Hum Pathol 1984;15:839-52.

11. Mitsui F, Aikata H, Hashimoto Y, et al. A first case of hepatic angiosarcoma treated with recombinant interleukin-2. Hiroshima J Med Sci 2011;60:91-6.
12. Sumrall A, Fredericks R, Berthold A, et al. Lenalidomide stops progression of multifocal epithelioid hemangioendothelioma including intracranial disease. J Neurooncol 2010;97:275-7.

13. Soape MP, Verma R, Payne JD, et al. Treatment of Hepatic Epithelioid Hemangioendothelioma: Finding Uses for Thalidomide in a New Era of Medicine. Case Rep Gastrointest Med 2015;2015:326795.

14. Mehrabi A, Kashfi A, Schemmer P, et al. Surgical treatment of primary hepatic epithelioid hemangioendothelioma. Transplantation 2005;80:S109-12.

15. St Peter SD, Moss AA, Huettl EA, et al. Chemoembolization followed by orthotopic liver transplant for epithelioid hemangioendothelioma. Clin Transplant 2003;17:549-53.

16. Lerut JP, Orlando G, Sempoux C, et al. Hepatic haemangioendothelioma in adults: excellent outcome following liver transplantation. Transpl Int 2004;17:202-7.

17. Cascarón MF, Dietrich A, Quesada E, et al. Nutritional management of an adult patient before, during and after liver partition (ALPPS) for diffuse hepatic epithelioid hemangioendothelioma. In: Carrera M, Gil A, Martinez JA. editors. IUNS. 21st International Congress of Nutrition. Buenos Aires, Argentina, October 15-20, 2017 : Abstracts. Ann Nutr Metab 2017;71:942.

18. Soubrane O, Sepulveda A, Schwarz L, et al. EPL-29: Laparoscopic associated liver partition and portal vein ligation for staged hepatectomy (ALPPS) for bilobar epithelioid hemangioendothelioma. HPB (Oxford) 2014;16:714.

19. Schnitzbauer AA, Lang SA, Goessmann H, et al. Right portal vein ligation combined with in situ splitting induces rapid left lateral liver lobe hypertrophy enabling 2 -staged extended right hepatic resection in small-for-size settings. Ann Surg 2012;255:405-14.

20. Schelotto PB, Gondolesi G. Laparoscopy in ALPPS Procedure: When We Can Do It? Ann Surg 2017;265:e30-1.

\section{doi: $10.21037 / \operatorname{tgh}-20-310$}

Cite this article as: Kawka M, Mak S, Qiu S, Gall TMH, Jiao LR. Hepatic epithelioid hemangioendothelioma (HEHE) rare vascular malignancy mimicking cholangiocarcinoma: a case report. Transl Gastroenterol Hepatol 2022;7:42. 